ELECTRONIC RESEARCH ANNOUNCEMENTS OF THE AMERICAN MATHEMATICAL SOCIETY

Volume 4, Pages 48-55 (July 31, 1998)

S $1079-6762(98) 00046-8$

\title{
THE INCIPIENT INFINITE CLUSTER IN HIGH-DIMENSIONAL PERCOLATION
}

\author{
TAKASHI HARA AND GORDON SLADE
}

(Communicated by Klaus Schmidt)

\begin{abstract}
We announce our recent proof that, for independent bond percolation in high dimensions, the scaling limits of the incipient infinite cluster's two-point and three-point functions are those of integrated super-Brownian excursion (ISE). The proof uses an extension of the lace expansion for percolation.
\end{abstract}

\section{INTRODUCTION}

Percolation has received much attention in mathematics and in physics, as a simple model of a phase transition. To describe the phase transition, associate to each bond $\{x, y\}\left(x, y \in \mathbb{Z}^{d}\right.$, separated by unit Euclidean distance) a Bernoulli random variable $n_{\{x, y\}}$ taking the value 1 with probability $p$ and the value 0 with probability $1-p$. These random variables are independent, and $p$ is a control parameter in $[0,1]$. A bond $\{x, y\}$ is said to be occupied if $n_{\{x, y\}}=1$, and vacant if $n_{\{x, y\}}=0$. The control parameter $p$ is thus the density of occupied bonds in the infinite lattice $\mathbb{Z}^{d}$. The percolation phase transition is the fact that, for $d \geq 2$, there is a critical value $p_{c}=p_{c}(d) \in(0,1)$, such that for $p<p_{c}$ there is with probability 1 no infinite connected cluster of occupied bonds, whereas for $p>p_{c}$ there is with probability 1 a unique infinite connected cluster of occupied bonds (percolation occurs).

There is now a considerable mathematical understanding of the subcritical regime $p<p_{c}$ and the supercritical regime $p>p_{c}[13,14,20,21]$, but the rich universal behaviour at the critical point $p=p_{c}$ remains largely the province of physics rather than mathematics. In particular, there is still no general proof of the widely accepted statement that there is no infinite cluster when $p=p_{c}$. This has been proved only for $d=2[13]$ (and references therein), $d \geq 19[6,17,19]$, and for $d>6$ for sufficiently spread-out models $[6,17]$ of the type described below. We focus in this paper on the high-dimensional case, where the absence of percolation at $p_{c}$ has been established.

The percolation phase transition presents a picture where at $p_{c}$ there are extensive connections present, on all length scales, but no infinite cluster. However, the slightest increase in $p$ will lead to the formation of an infinite cluster. This inchoate

Received by the editors March 17, 1998 and, in revised form, May 20, 1998.

1991 Mathematics Subject Classification. Primary 82B43, 60K35.

Key words and phrases. Critical exponent, incipient infinite cluster, integrated super-Brownian excursion, percolation, scaling limit, super-Brownian motion.

(C) 1998 American Mathematical Society 
state of affairs at $p_{c}$ is often represented by an appeal to the notion of the "incipient infinite cluster."

The incipient infinite cluster has been defined in 2-dimensional models as an infinite cluster in $\mathbb{Z}^{2}$ constructed by an appropriate limiting process [22], or by introducing an inhomogeneity [9]. We will approach the incipient infinite cluster from a different perspective. Rather than attempting to construct an infinite object on the lattice, we instead take a scaling limit of increasingly large but finite clusters, at $p=p_{c}$. This involves shrinking the lattice spacing as a function of the cluster size $n$ in such a way as to produce a nontrivial random subset of the continuum $\mathbb{R}^{d}$, in the limit $n \rightarrow \infty$. This is analogous to the procedure by which Brownian motion on the time interval $[0,1]$ can be constructed as a limit of an increasingly long lattice random walk. The appropriate spatial scaling of the lattice is presumably $n^{-1 / D_{H}}$, where $D_{H}$ corresponds to the Hausdorff dimension of the incipient infinite cluster. For $d>6$, it is believed that $D_{H}=4$, and accordingly we will scale down the lattice spacing by $n^{1 / 4}$.

Let $x \in \mathbb{R}^{d}$ be fixed. We prove that in sufficiently high dimensions, the probability that a site $\left\lfloor x n^{1 / 4}\right\rfloor \in \mathbb{Z}^{d}$ is connected to the origin in a cluster of size $n$, corresponds, in the scaling limit $n \rightarrow \infty$, to the mean mass density function of integrated super-Brownian excursion (ISE) at $x$. This will be stated more precisely in Theorem 1 below. ISE is a random probability measure on $\mathbb{R}^{d}$, representing a continuous-time branching process in which branching occurs on all (arbitrarily short) length scales $[5,10]$, and forms a basic example in the theory of superprocesses. For $d>4$, the support of this random probability measure almost surely has Hausdorff dimension 4 [10]. The support of the ISE probability measure is almost surely a compact random subset of $\mathbb{R}^{d}$, but this corresponds to an infinite lattice object.

We also prove that in sufficiently high dimensions, the probability that the origin is connected to sites $\left\lfloor x n^{1 / 4}\right\rfloor$ and $\left\lfloor y n^{1 / 4}\right\rfloor\left(x, y \in \mathbb{R}^{d}\right)$ corresponds, in the scaling limit, to the ISE mean joint mass density at $(x, y)$. A precise statement will be given in Theorem 2 below.

We conjecture that the scaling limit of the incipient infinite cluster is ISE for all dimensions $d>6$. The upper critical dimension 6 has long been identified as the dimension above which the behaviour of percolation models near $p_{c}$ no longer exhibits the dimension-dependence typical of lower dimensions, and adopts behaviour associated with percolation on trees. Our results mentioned above for the two- and three-point functions are restricted to sufficiently high dimensions (we have not computed how high is sufficient), rather than to $d>6$, in part because we use an expansion method, the lace expansion, for which the inverse dimension is the small parameter ensuring convergence.

There is an alternate small parameter that has been used in lace expansion methods in the past, which removes the need for the spatial dimension to serve as a small parameter, and allows for a control of all dimensions above the upper critical dimension. This involves the introduction of spread-out models, in which the nearest-neighbour bonds used above are enriched to a set of bonds of the form $\{x, y\}$ with $0<\|x-y\| \leq L$, where the norm is, for example, the supremum norm. Again we define Bernoulli random variables $n_{\{x, y\}}$ for each bond, just as was done for the nearest-neighbour model. We take $L$ large, with $L^{-1}$ serving as a small parameter to make the lace expansion converge. The conventional wisdom (still unproved in general), and an assertion of the hypothesis of universality, is that in 
any dimension $d$ the spread-out models have identical critical behaviour for all finite $L \geq 1$, and for any choice of norm which respects the lattice symmetries. This is analogous to the fact that diverse lattice random walks have the same Brownian scaling limit.

At present, our method is not adequate to prove that the scaling limits of the probability of a connection of two points, or three points, is the corresponding ISE density for sufficiently spread-out models in all dimensions $d>6$. This is due to a difficulty, associated with the fact that ISE has self-intersections in dimension less than 8 , which currently prevents us from handling dimensions 7 and 8 in such detail. However, as we will indicate below in Theorem 3, we are able to give some indication that ISE may be relevant to the scaling limit of the incipient infinite cluster, for sufficiently spread-out models in all dimensions $d>6$.

The study of the scaling limit of the incipient infinite cluster is basic in the analysis of the continuum limit of critical percolation. Above six dimensions, work in this direction has been carried out by Aizenman [1] (see also [7, 8]). Aizenman's results, which are based on the assumption (as yet, unproved) that at $p_{c}$ the probability of a distant site $x$ being connected to the origin is comparable to $|x|^{2-d}$, are complementary to ours. In particular, a picture is described in [1] for percolation on a lattice with $d>6$ and with small spacing $a$, where in a window of fixed size in the continuum, the largest clusters have size of order $a^{-4}$ and there are of order $a^{6-d}$ clusters of this size. Our results suggest that for $d>6$ a cluster of size $n=a^{-4}$ in a lattice with spacing $a=n^{-1 / 4}$ will typically be an ISE cluster, in general rescaled to an unnormalized measure, in the scaling limit.

\section{The Results}

Consider independent bond percolation (nearest-neighbour or spread-out) with $p=p_{c}$. Let $C(0)$ denote the random set of sites connected to 0 by a path consisting of occupied bonds, and let $|C(0)|$ denote its cardinality. Let

$$
\tau(x ; n)=P(C(0) \ni x,|C(0)|=n)
$$

denote the probability at $p_{c}$ that the origin is connected to $x$ by a cluster containing $n$ sites. Then

$$
q_{n}(x)=\frac{\tau(x ; n)}{\sum_{x \in \mathbb{Z}^{d}} \tau(x ; n)}=\frac{\tau(x ; n)}{n P(|C(0)|=n)}
$$

defines a probability measure on $\mathbb{Z}^{d}$ proportional to the conditional probability at $p_{c}$ that a cluster of size $n$ contains $x$.

For $k \in \mathbb{R}^{d}$, define

$$
\hat{A}^{(2)}(k)=\int_{0}^{\infty} t e^{-t^{2} / 2} e^{-k^{2} t / 2} d t .
$$

This is the Fourier integral transform of the mean mass density function

$$
A^{(2)}(x)=\int_{0}^{\infty} t e^{-t^{2} / 2}(2 \pi t)^{-d / 2} e^{-x^{2} / 2 t} d t
$$

of ISE; for a discussion of this formula, see [5, 11, 4, 23]. The following theorem shows that in the scaling limit, the two-point function of the incipient infinite cluster is the two-point function of ISE, in sufficiently high dimensions. In its statement, we use the discrete Fourier transform $\hat{f}(k)=\sum_{x \in \mathbb{Z}^{d}} f(x) e^{i k \cdot x}\left(k \in[-\pi, \pi]^{d}\right)$, for a summable function $f$ on $\mathbb{Z}^{d}$. 
Theorem 1. Fix $k \in \mathbb{R}^{d}$ and any $\epsilon \in\left(0, \frac{1}{2}\right)$. For the nearest-neighbour model with $d$ sufficiently large and $p=p_{c}$, there are $d$-dependent positive constants $C, D$ such that

$$
\hat{\tau}\left(k D^{-1} n^{-1 / 4} ; n\right)=\frac{C}{\sqrt{8 \pi n}} \hat{A}^{(2)}(k)\left[1+O\left(n^{-\epsilon}\right)\right] .
$$

In particular,

$$
P(|C(0)|=n)=\frac{1}{n} \hat{\tau}(0 ; n)=\frac{C}{\sqrt{8 \pi} n^{3 / 2}}\left[1+O\left(n^{-\epsilon}\right)\right],
$$

and

$$
\lim _{n \rightarrow \infty} \hat{q}_{n}\left(k D^{-1} n^{-1 / 4}\right)=\hat{A}^{(2)}(k) .
$$

Equation (2.6) asserts that $\delta=2$, where $\delta$ is the critical exponent in the conjectured relation $P(|C(0)|=n) \approx n^{-1-1 / \delta}$. Equation (2.7) can be interpreted as asserting that in the scaling limit the distribution of a site $\left\lfloor x D n^{1 / 4}\right\rfloor$ in the cluster of the origin, conditional on the cluster being of size $n$, is the distribution of a point in ISE.

We now consider the three-point function. Let $\tau^{(3)}(x, y ; n)$ denote the probability, at $p_{c}$, that the origin is connected to $x$ and $y$ and that the cluster of the origin contains exactly $n$ sites. For $k, l \in[-\pi, \pi]^{d}$, define

$$
\hat{\tau}^{(3)}(k, l ; n)=\sum_{x, y \in \mathbb{Z}^{d}} \tau^{(3)}(x, y ; n) e^{i k \cdot x} e^{i l \cdot y} .
$$

We define a probability measure on $\mathbb{Z}^{d} \times \mathbb{Z}^{d}$ by

$$
q_{n}^{(3)}(x, y)=\frac{\tau^{(3)}(x, y ; n)}{\sum_{x, y \in \mathbb{Z}^{d}} \tau^{(3)}(x, y ; n)}=\frac{\tau^{(3)}(x, y ; n)}{n^{2} P(|C(0)|=n)} .
$$

For $k, l \in \mathbb{R}^{d}$, let $\hat{A}^{(3)}(k, l)$ denote the Fourier transform of the ISE three-point function:

$$
\hat{A}^{(3)}(k, l)=\int_{0}^{\infty} \int_{0}^{\infty} \int_{0}^{\infty}\left(\sum_{j=1}^{3} t_{j}\right) e^{-\left(\sum_{j=1}^{3} t_{j}\right)^{2} / 2} e^{-\left[(k+l)^{2} t_{1}+k^{2} t_{2}+l^{2} t_{3}\right] / 2} d t_{1} d t_{2} d t_{3} .
$$

Equation (2.10) differs from the formulas of $[5,11]$ in that here we have not fixed the location of the internal branch point. The next theorem shows that in the scaling limit, the three-point function of the incipient infinite cluster corresponds to that of ISE, in high dimensions. The constants $C, D$ in the theorem are the same as those appearing in Theorem 1.

Theorem 2. Fix $k \in \mathbb{R}^{d}$ and any $\epsilon \in\left(0, \frac{1}{2}\right)$. For the nearest-neighbour model with $d$ sufficiently large and $p=p_{c}$,

$$
\hat{\tau}^{(3)}\left(k D^{-1} n^{-1 / 4}, l D^{-1} n^{-1 / 4} ; n\right)=\frac{C}{\sqrt{8 \pi}} n^{1 / 2} \hat{A}^{(3)}(k, l)\left[1+O\left(n^{-\epsilon}\right)\right] .
$$

In particular,

$$
\lim _{n \rightarrow \infty} \hat{q}_{n}^{(3)}\left(k D^{-1} n^{-1 / 4}, l D^{-1} n^{-1 / 4}\right)=\hat{A}^{(3)}(k, l)
$$


Note that for $(k, l)=(0,0)$, Theorem 2 follows immediately from Theorem 1 , since $\hat{\tau}^{(3)}(0,0 ; n)=n^{2} P(|C(0)|=n)$ and $\hat{A}^{(3)}(0,0)=1$.

As mentioned in Section 1, for sufficiently spread-out models in dimensions $d>6$, we have a weaker result. In preparation for this, we define a generating function $\Lambda_{z}(k)$ and coefficients $\lambda_{n}(k)$ by

$$
\Lambda_{z}(k)=\frac{1}{k^{2}+2^{3 / 2} \sqrt{1-z}}=\sum_{n=0}^{\infty} \lambda_{n}(k) z^{n} .
$$

The square root has branch cut $[1, \infty)$, and the branch with $\sqrt{1-z}$ positive for $z \in(-\infty, 1)$ is chosen. The power series has radius of convergence 1 . By Cauchy's theorem,

$$
\lambda_{n}(k)=\frac{1}{2 \pi i} \oint_{\Gamma} \Lambda_{z}(k) \frac{d z}{z^{n+1}},
$$

where $\Gamma$ is a circle centred at the origin, of any radius less than 1 . An elementary computation extending [11, Lemma 1] shows that

$$
\lambda_{n}\left(k n^{-1 / 4}\right)=\frac{1}{\sqrt{8 \pi n}} \hat{A}^{(2)}(k)+O\left(n^{-3 / 2}\right),
$$

demonstrating a link between ISE and the generating function $\Lambda_{z}(k)$. As we will explain in Section 3, this link is a key element in the proof of Theorems 1 and 2 .

We define the generating function

$$
\tau_{z}(x)=\sum_{n=1}^{\infty} \tau(x ; n) z^{n}, \quad|z| \leq 1 .
$$

The parameter $z$ is a complex variable. It is not hard to show that the Fourier transform $\hat{\tau}_{z}(k)=\sum_{x} \tau_{z}(x) e^{i k \cdot x}$ exists for $|z|<1$. When $z \in[0,1]$, it is traditional to write $z=e^{-h}$, with $h$ playing the role of a magnetic field, but since here $z$ is in general complex, we will not adopt this notation. The following theorem shows that the generating function $\Lambda_{z}(k)$ is relevant for sufficiently spread-out percolation models in all $d>6$, and provides a statement, in that context, linking the incipient infinite cluster to ISE.

Theorem 3. For any $d>6$, there are positive constants $C, D$ (depending on $d, L$ ) and an $L_{0}(d)$ (large), such that for $L \geq L_{0}(d), k \in[-\pi, \pi]^{d}$ and $z \in[0,1)$,

$$
\hat{\tau}_{z}(k)=C \Lambda_{z}(D k)[1+\epsilon(z, k)]
$$

where $|\epsilon(z, k)| \leq \epsilon_{1}(z)+\epsilon_{2}(k)$ with $\lim _{z \rightarrow 1} \epsilon_{1}(z)=\lim _{k \rightarrow 0} \epsilon_{2}(k)=0$.

The control of the limit $z \rightarrow 1$ provides a somewhat different statement from the infra-red bound of [17] that $\eta=0$, while taking $k=0$ improves on the statement $\delta=2$ from $[6,17]$. The critical exponents $\eta$ and $\delta$ appear in the relations $\hat{\tau}_{1}(k) \approx$ $k^{-2+\eta}$ as $k \rightarrow 0$, and $\hat{\tau}_{z}(0) \approx(1-z)^{1 / \delta-1}$ as $z \uparrow 1$, which are conjectured to hold in general dimensions, with $d$-dependent values for the exponents when $d<6$.

The proof of Theorem 3 is given in [15], and Theorems 1 and 2 are proved in [16]. 


\section{THE METHOD}

The method of proof of Theorem 1 involves showing that in high dimensions it is possible to write, for $|z|<1$,

$$
\hat{\tau}_{z}(k)=\frac{C}{D^{2} k^{2}+2^{3 / 2}(1-z)^{1 / 2}}+E_{z}(k)=C \Lambda_{z}(D k)+E_{z}(k),
$$

with $E_{z}(k)=\sum_{n=1}^{\infty} e_{n}(k) z^{n}$ and $\left|e_{n}\left(k D^{-1} n^{-1 / 4}\right)\right| \leq O\left(n^{-\epsilon-1 / 2}\right)$. This is sufficient, in view of (2.15). The leading behaviour of (3.1) corresponds to the mean-field critical exponents $\delta=2$ and $\eta=0$. Theorem 3 is in the same spirit as (3.1), but does not involve complex variables or power-law error estimates, and is easier to establish.

For Theorem 2, we define $\hat{\tau}_{z}^{(3)}(k, l)=\sum_{n=1}^{\infty} \hat{\tau}^{(3)}(k, l ; n) z^{n}$ and show that

$$
\hat{\tau}_{z}^{(3)}(k, l)=4 C \Lambda_{z}(D(k+l)) \Lambda_{z}(D k) \Lambda_{z}(D l)+E_{z}^{(3)}(k, l),
$$

with $E_{z}^{(3)}(k, l)=\sum_{n=1}^{\infty} e_{n}^{(3)}(k, l) z^{n}$ and $\left|e_{n}^{(3)}\left(k D^{-1} n^{-1 / 4}, l D^{-1} n^{-1 / 4}\right)\right| \leq O\left(n^{-\epsilon+1 / 2}\right)$. An elementary contour integration, as in (2.14) but for a product of $\Lambda_{z}$ 's, then gives the theorem. Combining (3.1) and (3.2), to leading order the three-point function obeys

$$
\hat{\tau}_{z}^{(3)}(k, l)=4 C^{-2} \hat{\tau}_{z}(k+l) \hat{\tau}_{z}(k) \hat{\tau}_{z}(l)+\text { error. }
$$

This factorization corresponds to an effective independence that is discussed further below, and is in the spirit of a conjecture of [3].

Our proof of (3.1) involves an analysis of the $z$-derivative of $\hat{\tau}_{z}(k)$. This leads naturally to the study of $\hat{\tau}_{z}^{(3)}$, since

$$
\begin{aligned}
z \frac{d}{d z} \hat{\tau}_{z}(k) & =\sum_{x \in \mathbb{Z}^{d}} e^{i k \cdot x} \sum_{n=1}^{\infty} n \tau(x ; n) z^{n} \\
& =\sum_{x, y \in \mathbb{Z}^{d}} e^{i k \cdot x} \sum_{n=1}^{\infty} \tau^{(3)}(x, y ; n) z^{n}=\hat{\tau}_{z}^{(3)}(k, 0) .
\end{aligned}
$$

The study of the three-point function is thus central for our method.

Equation (3.3) can be understood in terms of the schematic figure

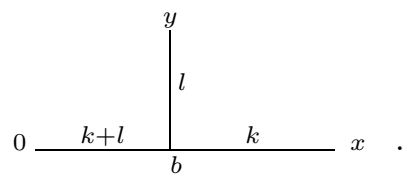

The connections in the figure represent edge-disjoint connections by occupied paths in the cluster of the origin, and the branch point $b$ is not uniquely defined. In high dimensions, the three parts of the diagram, corresponding to the connections $0 \rightarrow b, b \rightarrow x, b \rightarrow y$, can be regarded, to leading order, as effectively independent. This independence is not exact, but rather correction terms involving the triangle diagram of [3], and related diagrams, give rise to the renormalized vertex factor $4 C^{-2}$ appearing in (3.3).

The establishment of this effective independence is at the heart of the lace expansion method of [17] (see also [19, 24]), and allows for the demonstration of the 
independence of the connections $0 \rightarrow b$ and $b \rightarrow x$, without the presence of the variable $y$. A second expansion is required to demonstrate the effective independence of the connection $b \rightarrow y$. This is technically involved, but is conceptually similar to the first expansion. Such a double expansion has been carried out previously in the analysis of lattice trees in high dimensions [18], and was used to prove results similar to Theorems 1 and 2 in that context $[12,11]$.

The previous development of the lace expansion for percolation was restricted to the case $z=1$ [17]. Working with general $z$ provides new difficulties to overcome. For general $z$, we generate the expansion using a probabilistic interpretation, valid for positive $z$, that was used, e.g., in [2]. In this interpretation, the sites in $\mathbb{Z}^{d}$ are declared to be "not green" with probability $z \in[0,1]$ and "green" with probability $1-z$. The site variables are independent, and independent of the bond variables. Then, for $z \in[0,1], \tau_{z}(x)$ can be interpreted as the probability that the origin is connected to $x$ but is not connected to any green site. This green-free condition on the two-point function necessitates major revision of the lace expansion methodology. The expansion can be extended from positive to complex $z$ via analyticity.

\section{The Backbone}

ISE can be understood as a process evolving in time, and it is of interest to interpret our results in terms of a time variable. For this, we introduce the notion of the backbone of a cluster containing two sites $x$ and $y$. We define the backbone, which depends on $x, y$, to consist of those sites $u \in C(x)$ for which there are edge-disjoint paths consisting of occupied bonds from $x$ to $u$ and from $u$ to $y$. The backbone thus consists of connections from $x$ to $y$, with all "dangling ends" removed.

We believe it would be of interest to attempt to extend our methods, in combination with the methods of [12], to prove that (for high dimensions) in a cluster of size $n$, a backbone joining sites $\left\lfloor x n^{1 / 4}\right\rfloor$ and $\left\lfloor y n^{1 / 4}\right\rfloor\left(x, y \in \mathbb{R}^{d}\right)$ typically consists of $O\left(n^{1 / 2}\right)$ sites and converges in the scaling limit to a Brownian path, with the Brownian time variable corresponding to distance along the backbone. Such a study has not been carried out for percolation, but an analogous result has been proved for high-dimensional lattice trees in [12, Theorem 1.2]. In this interpretation, the integration variables $t$ and $t_{i}$ appearing in $\hat{A}^{(2)}(k)$ and $\hat{A}^{(3)}(k)$ correspond to time intervals for scaling limits of backbone paths.

The concept of the backbone is relevant for an understanding of the value 6 of the upper critical dimension. In the original lace expansion for percolation [17], as in the analysis involving the triangle condition in [3], the leading behaviour corresponds to

neglecting intersections between a backbone and a percolation cluster. Considering the backbone to correspond to a 2-dimensional Brownian path, and the cluster to correspond to a 4-dimensional ISE cluster, intersections will generically not occur above $2+4=6$ dimensions. This points to $d=6$ as the upper critical dimension.

\section{ACKNOWLEDGEMENTS}

This work was supported in part by NSERC, and was carried out in part while both authors were visiting the University of British Columbia in 1997 and Microsoft Research in 1998. The work of G.S. was also supported in part by a 1996 Invitation Fellowship of the Japan Society for the Promotion of Science. We thank Michael Aizenman and Ed Perkins for stimulating conversations and correspondence. 


\section{REFERENCES}

[1] M. Aizenman, On the number of incipient spanning clusters, Nucl. Phys. B [FS] 485 (1997), 551-582. CMP 97:07

[2] M. Aizenman and D.J. Barsky, Sharpness of the phase transition in percolation models, Commun. Math. Phys. 108 (1987), 489-526. MR 88c:82026

[3] M. Aizenman and C.M. Newman, Tree graph inequalities and critical behavior in percolation models, J. Stat. Phys. 36 (1984), 107-143. MR 86h:82045

[4] D. Aldous, The continuum random tree III, Ann. Probab. 21 (1993), 248-289. MR 94c:60015

[5] _ Tree-based models for random distribution of mass, J. Stat. Phys. 73 (1993), 625641. MR 94k:60130

[6] D.J. Barsky and M. Aizenman, Percolation critical exponents under the triangle condition, Ann. Probab. 19 (1991), 1520-1536. MR 93b:60224

[7] C. Borgs, J.T. Chayes, H. Kesten, and J. Spencer, The birth of the infinite cluster: finite size scaling in percolation. In preparation.

[8] _ Uniform boundedness of critical crossing probabilities implies hyperscaling. In preparation.

[9] J.T. Chayes, L. Chayes, and R. Durrett, Inhomogeneous percolation problems and incipient infinite clusters, J. Phys. A: Math. Gen. 20 (1987), 1521-1530. MR 88k:82089

[10] D. Dawson and E. Perkins, Measure-valued processes and renormalization of branching particle systems, Stochastic Partial Differential Equations: Six Perspectives (R. Carmona and B. Rozovskii, eds.), AMS Math. Surveys and Monographs. To appear.

[11] E. Derbez and G. Slade, Lattice trees and super-Brownian motion, Canad. Math. Bull. 40 (1997), 19-38. CMP 97:11

[12] _ , The scaling limit of lattice trees in high dimensions, Commun. Math. Phys. 193 (1998), 69-104. CMP 98:11

[13] G. Grimmett, Percolation, Springer, Berlin, 1989. MR 90j:60109

[14] _ Percolation and disordered systems (St. Flour lecture notes, 1996), Lecture Notes in Math., vol. 1665, Springer, Berlin, 1997. CMP 98:06

[15] T. Hara and G. Slade, The scaling limit of the incipient infinite cluster in high-dimensional percolation. I. Critical exponents. In preparation.

[16] - The scaling limit of the incipient infinite cluster in high-dimensional percolation. II. Integrated super-Brownian excursion. In preparation.

[17] _ Mean-field critical behaviour for percolation in high dimensions, Commun. Math. Phys. 128 (1990), 333-391. MR 91a:82037

[18] _ The number and size of branched polymers in high dimensions, J. Stat. Phys. 67 (1992), 1009-1038. MR 93c:82075

[19] _ Mean-field behaviour and the lace expansion, Probability and Phase Transition (Dordrecht) (G. Grimmett, ed.), Kluwer, 1994. MR 95d:82033

[20] B.D. Hughes, Random walks and random environments, vol. 2: Random Environments, Oxford University Press, New York, 1996. MR 98d:60139

[21] H. Kesten, Percolation theory for mathematicians, Birkhäuser, Boston, 1982. MR 84i:60145

$[22]$, The incipient infinite cluster in two-dimensional percolation, Probab. Th. Rel. Fields 73 (1986), 369-394. MR 88c:60196

[23] J.-F. Le Gall, The uniform random tree in a Brownian excursion, Probab. Th. Rel. Fields 96 (1993), 369-383. MR 94e:60073

[24] N. Madras and G. Slade, The self-avoiding walk, Birkhäuser, Boston, 1993. MR 94f:82002

Department of Applied Physics, Tokyo Institute of Technology, Oh-Okayama, MeguroKU, TOKYO 152, JAPAN

E-mail address: hara@ap.titech.ac.jp

Department of Mathematics and Statistics, McMaster University, Hamilton, ON, CANADA L8S $4 \mathrm{~K} 1$

E-mail address: slade@mcmaster.ca 